\title{
PULSED ELECTROMAGNETIC FIELDS SYNERGIZE WITH GRAPHENE TO ENHANCE DENTAL PULP STEM CELL-DERIVED NEUROGENESIS BY SELECTIVELY TARGETING TRPC1 CHANNELS
}

\author{
T.T. Madanagopal ${ }^{1,2,3, \$}$, Y.K. Tai ${ }^{4,5,8}$, S.H. Lim ${ }^{6}$, C.H.H. Fong ${ }^{4,5}$, T. Cao ${ }^{1}$, V. Rosa ${ }^{1,7}$ \\ and A. Franco-Obregón ${ }^{4,5,8,9,10, *}$ \\ ${ }^{1}$ Faculty of Dentistry, National University of Singapore, Singapore \\ ${ }^{2}$ Molecular Neuropsychiatry \& Development (MiND) Lab, Campbell Family Mental Health Research \\ Institute, Centre for Addiction and Mental Health, Toronto, ON, Canada \\ ${ }^{3}$ Institute of Medical Science, University of Toronto, Toronto, ON, Canada \\ ${ }^{4}$ Department of Surgery, Yong Loo Lin School of Medicine, National University of Singapore, Singapore \\ ${ }^{5}$ Biolonic Currents Electromagnetic Pulsing Systems Laboratory, BICEPS, \\ National University of Singapore, Singapore \\ ${ }^{6}$ Department of Pharmacy, National University of Singapore, Singapore \\ ${ }^{7}$ Craniofacial Research and Innovation Center, National University of Singapore, Singapore \\ ${ }^{8}$ Department of Physiology, Yong Loo Lin School of Medicine, \\ National University of Singapore, Singapore \\ ${ }^{9}$ Institute for Health Innovation \& Technology, iHealthtech, National University of Singapore, Singapore \\ ${ }^{10}$ Healthy Longevity Translational Research Programme, Yong Loo Lin School of Medicine, \\ NUS, Singapore \\ $\S$ These authors contributed equally to the work
}

\begin{abstract}
Conventional root canal treatment replaces the infected pulp with defined materials. Alternative cell-based tissue engineering strategies aim to regenerate a fully functional pulp within the root canal. Despite recent advances in this area, however, the regeneration of an innervated pulp remains a major challenge in the field. Both graphene (2DG) and pulsed electromagnetic fields (PEMFs) independently have been shown to promote diverse cellular developmental programs. The present study showed that 2DG promoted the neurogenic induction of human dental pulp stem cells (hDPSCs) by upregulating and accelerating the expression of mature neuronal markers. Notably, 2DG induced the highest expression of transient receptor potential canonical cation channel type 1 (TRPC1) during early neurogenesis. As brief PEMF exposure promotes in vitro differentiation by activating a TRPC1-mitochondrial axis, an opportunity to combine 2DG with developmentally targeted PEMF exposure for synergistic effects was realizable. Neurogenic gene expression, neurotransmitter release, and reactive oxygen species (ROS) production were greatly enhanced by a brief (10 min) and low amplitude ( $2 \mathrm{mT})$ PEMF exposure timed to coincide with the highest TRPC1 expression from hDPSCs on 2DG. In contrast, hDPSCs on glass were less responsive to PEMF exposure. The capacity of PEMFs to promote neurogenesis was precluded by the administration of penicillin/streptomycin, mirroring previous studies demonstrating that aminoglycoside antibiotics block TRPC1-mediated calcium entry and verifying the contribution of TRPC1 in this form of magnetoreception. Hence, graphene created a more conducive environment for subsequent PEMF-stimulated neurogenic induction of hDPSCs through their mutual capacity to activate TRPC1with subsequent ROS production.
\end{abstract}

Keywords: Pulsed electromagnetic fields, mitohormesis, tissue engineering, nanomaterial, pulp regeneration.

*Address for correspondence: Alfredo Franco-Obregón, Department of Surgery, Yong Loo Lin School of Medicine, National University of Singapore, NUHS Tower Block, Level 8, IE Kent Ridge Road, Singapore, 119228 Singapore.

Telephone number: +65 67778427 Email: suraf@nus.edu.sg

Copyright policy: This article is distributed in accordance with Creative Commons Attribution Licence (http://creativecommons.org/licenses/by-sa/4.0/). 


\section{List of Abbreviations}

$2 \mathrm{DG}$

\section{$\mathrm{ACh}$ \\ BDNF \\ BMP \\ CACNA1C}

CACNA2D2 calcium voltage-gated channel auxiliary subunit $\alpha 2 \delta 2$

$\begin{array}{ll}\text { cAMP } & \begin{array}{l}\text { cyclic adenosine monophosphate } \\ \text { choline acetyltransferase }\end{array} \\ \text { ChT } & \text { high-affinity choline transporter } \\ \text { DMEM } & \begin{array}{l}\text { Dulbecco's-modified Eagle medium } \\ \text { enolase }\end{array} \\ \text { ENO-2 } & \text { focal adhesion kinase } \\ \text { FAK } & \text { fetal bovine serum } \\ \text { FBS } & \begin{array}{l}\text { full width at half maximum } \\ \text { glyceraldehyde-3-phosphate } \\ \text { FWHM }\end{array} \\ \text { GAPDH } & \begin{array}{l}\text { glial-cell-line-derived neurotrophic } \\ \text { factor }\end{array}\end{array}$

HB-9 homeobox gene Hb9

hDPSCs human dental pulp stem cells

IGF-1 insulin-like growth factor 1

ISL ISL LIM homeobox

MAPK mitogen-activated protein kinase

MSC mesenchymal stem cell

MYH myosin heavy chain

MTS 3-(4,5-dimethylthiazol-2-yl)-5-(3-

carboxymethoxyphenyl)-2-(4-

sulfophenyl)-2H tetrazolium, inner

salt

NFAT nuclear factor of activated T-cells

NF-H neurofilament heavy

NF-M neurofilament medium

OLIGO2 oligodendrocyte transcription factor 2
PCL

PCNA

PEMF

PLGA

PS

RT-PCR

ROS

SMN

TRP

TRPC

TRPM

TRPV

TUB-3

VAChT

polycaprolactone

proliferating cell nuclear antigen

pulsed electromagnetic field

poly(lactic-co-glycolic acid)

penicillin/streptomycin

real-time polymerase chain reaction

reactive oxygen species

survival motor neuron

transient receptor potential

transient receptor potential cation

channel subfamily C

transient receptor potential cation

channel subfamily $\mathrm{M}$

transient receptor potential cation

channel subfamily $\mathrm{V}$

tubulin $\alpha-3$ chain

vesicular acetylcholine transporter

\section{Introduction}

During a routine root canal treatment, the injured dental pulp is replaced with defined biomaterials, resulting in a pulpless tooth that is incapable of sensing inflammation from microbial ingress (Rosa et al., 2011). Hence, an ongoing objective has been the development of cell-based strategies aimed at the tissue engineering of a fully functional dental pulp. Although headway has been made in the tissue engineering of dental pulp with a well-organized matrix and vascular network (Rosa et al., 2013; Sakai et al., 2010; Xie et al., 2017b), the establishment of a sufficiently innervated dental pulp to reinstate sensory and protective functions against injuries remains a major challenge (Moussa and Aparicio, 2018). MSCs have been successfully differentiated into neuronal precursors, expressing mature neuronal markers and neuronal functionality (Fričová et al., 2020; Papaccio et al., 2019). The human dental pulp harbors hDPSCs that are mesenchymal in phenotype and comparable to bone-marrow-derived MSCs in the breadth of their expression profile of over 4,000 genes (Shi et al., 2001), including the MSCs markers CD29, CD44, CD73, CD90, CD105, CD117, CD146 and Stro-1 (Martens et al., 2012; Rosa et al., 2016). Moreover, hDPSCs can be readily obtained from routinely extracted third molars under local anesthesia, at a relatively low cost and without producing esthetic damage, presenting a significant advantage over the isolation protocols of other stem cell classes [for instance, the more invasive aspiration protocols required for bone marrow stem cells isolation (Gronthos et al., 2000; Rosa et al., 2012)]. hDPSCs derive from the embryonic neural crest and can be differentiated into either odontoblastic or neuronal-like cell lineages (Gronthos et al., 2000; Madanagopal et al., 2020). Nonetheless, neurogenic induction typically requires the use of expensive chemical cocktails that are commonly applied for extended periods of up to 7 weeks ( $\mathrm{Li}$ et al., 2008; Wada et al., 2009). Hence, the development of strategies to induce faster and more efficient neurogenic differentiation of hDPSCs at a lower cost are of great clinical interest.

Biomaterials such as PLGA and PCL can support the neurogenic differentiation of stem cells (Bahrami et al., 2017; Ebrahimi-Barough et al., 2015). However, such polymers release inflammatory by-products during their spontaneous degradation $\left(\mathrm{O}^{\prime}\right.$ Brien, 2011; Rosa et al., 2012). Hence, the development of biocompatible materials that can promote neuronal differentiation with low cytotoxicity is an active area of research. Graphene is a single atomic sheet of conjugated sp2 carbon atoms possessing a unique combination of physical, mechanical, and electrical properties conducive to stem cell differentiation (Luong-Van et al., 2020). Graphene films and scaffolds support the attachment and proliferation of various progenitor cell classes without signs of toxicity or material degradation (Kalbacova et al., 2010; Li et al., 2015; Li et al., 2013; Xie et al., 2015; Xie et al., 2019). Graphene substrates can also promote the differentiation of MSCs into osteoblasts, adipocytes, or neurons as well as facilitate neurite guidance and outgrowth (Dubey et al., 2020; Guo et al., 2016; Kim 
et al., 2015; Xie et al., 2015). Although hDPSCs have been differentiated into an odontoblastic-like lineage (Gronthos et al., 2000; Xie et al., 2017b), hDPSCs on graphene are less likely to adopt this phenotype (Xie et al., 2017a), creating an opportunity to fully harness the reported neurogenic attributes of graphene in hDPSCs (Madanagopal et al., 2020).

The molecular basis for the described capacity of graphene to promote stem cell differentiation into multiple lineages is yet to be fully understood (Luong-Van et al., 2020). On the one hand, it has been suggested that graphene enhances differentiation thanks to its ability to bind and accumulate proteins and growth factors, effectively increasing their local bioavailability (Lee et al., 2011). Apparently contradicting such a mechanism, withholding supplementation with exogenous induction factors, does not prevent graphene from promoting neurite outgrowth and elongation (Lee et al., 2018; Park et al., 2011), potentially reflecting endogenous trophic factor sequestration. On the other hand, graphene also augments cytoskeleton tension and upregulates the genetic and protein expressions of F-actin, FAK, vinculin, BMP-2, and MYH (Dubey et al., 2020; Li et al., 2015; Xie et al., 2019; Xie et al., 2015), implicating the involvement of mechanotransduction and indicating that graphene triggers cell differentiation through inherent biophysical cues (Luong-Van et al., 2020).

TRP channels are expressed in a wide variety of progenitor cell classes and differentiated tissues and respond to a broad modality of physical and chemical stimuli (Clapham, 2003). Diverse TRP channels are involved in the transduction of environmental stimuli into developmental responses governing proliferation, differentiation, or apoptosis. TRPC1 is the most ubiquitously expressed of all TRP channels (Jang et al., 2012) and is commonly expressed during early differentiation in diverse progenitor and stem cell classes (Crocetti et al., 2014; Golovina et al., 2001; Louis et al., 2008; Torossian et al., 2010). Low amplitude PEMFs can enhance in vitro MSC-derived chondrogenesis (Parate et al., 2017) and myoblastderived myogenesis (Yap et al., 2019) through their capacity to selectively activate TRPC1 channels. Specifically, PEMF-mediated activation of TRPC1 stimulates mitochondrial respiration and consequent ROS production upstream of eliciting mitochondrial survival adaptations following a process of magnetic mitohormesis (Yap et al., 2019). Indeed, vesicular delivery of TRPC1 alone to TRPC1 knockdown cells is sufficient to restore lost magnetosensitivity by reinstating mitochondrial responses to magnetic stimulation (Kurth et al., 2020). Similarly, PEMFs promote neurite outgrowth and differentiation in embryonic neural stem cells by recruiting the activity of TRPC1 channels (Ma et al., 2016). Quite recently, moreover, PEMF treatment also promotes the survival and proliferation of hDPSCs (Samiei et al., 2020). Nonetheless, PEMF-induced proliferation of hDPSCs required hours of treatment delivered over a week (Samiei et al., 2020), severely undermining their practical implementation in clinical environments. The present study showed that by combining PEMF intervention with 2DG, targeted to a time when TRPC1 channels are most developmentally expressed in vitro, it was possible to reduce the requisite PEMF exposure time to only $10 \mathrm{~min}$ applied once, while simultaneously accelerating and enhancing hDPSCderived neurogenesis.

\section{Materials and Methods}

Graphene production, transfer, and characterization Graphene (2DG) was produced by chemical vapordeposition using a custom-built furnace in a Class 1000 cleanroom facility, as previously described (Morin et al., 2017). Briefly, 2DG was coated on copper foils at $1,000^{\circ} \mathrm{C}$ using methane gas (16 SCCM, 30 min at $500 \mathrm{mTorr}$ ) and cooled to room temperature in a hydrogen-filled chamber. Then, the copper foil was etched in $1.5 \%$ ammonium persulfate for $8 \mathrm{~h}$ and the film transferred to deionized water for $24 \mathrm{~h}$. The transfer was completed by gently coating 2DG films onto glass coverslips (13 $\mathrm{mm}$ diameter), followed by incubation for $3 \mathrm{~h}$ in isopropanol. Then, samples were washed with deionized water and finally dried using nitrogen. 2DG samples were characterized by Raman spectroscopy (Raman Microscope CRM 200, Witec, Ulm, Germany; excitation laser source $532 \mathrm{~nm}$ ). An uncoated glass coverslip was used as a control (glass).

\section{hDPSC culture}

The use of human cells was approved by the NUS Institutional Review Board (NUS2094/2015). hDPSC (DPF003, AllCells, Quincy, MA, USA) were cultured in basal growth medium (DMEM, Invitrogen), supplemented with $10 \%$ FBS (Invitrogen) in the absence of PS. The expression of CD34, CD73, CD90, and CD105 was previously described (Rosa et al., 2016). All assays were performed using cells from passages 4 to 6 .

\section{Neuronal differentiation}

hDPSCs $\left(20 \times 10^{3}\right.$ cells $)$ were seeded onto 2 DG or glass (control substrate) and cultured with basal growth medium for $24 \mathrm{~h}$. After $24 \mathrm{~h}$, neurogenic differentiation was induced using a previously described protocol (Chang et al., 2014). The reagents used at different time points for neurogenic differentiation are listed in Table 1.

Expression of markers involved in neurogenic differentiation was assessed by qPCR, as described previously (Madanagopal et al., 2020). Briefly, hDPSCs were differentiated for $6 \mathrm{~d}$ on 2DG or glass before harvesting for total RNA (Purelink RNA Mini Kit, Invitrogen) and reverse transcribed to cDNA (iScript RT Supermix, Bio-Rad). The relative gene expression was quantified using the $\Delta \mathrm{Ct}$ method. Statistical significance in gene expression for hDPSCs on 2DG was calculated relative to respective gene expression from hDPSCs on glass using the Student's 
Table 1. Neurogenic differentiation protocol (* from LifeTech, ${ }^{,}$from Sigma-Aldrich).

\begin{tabular}{|c|c|c|}
\hline Time point & Medium & Components \\
\hline $24 \mathrm{~h}$ post seeding & Basal growth medium & $\begin{array}{c}\text { DMEM/F12 (489 mL) } \\
\text { N2* }(5 \mathrm{~mL}) \\
\text { Non-essential amino acids* }(5 \mathrm{~mL}) \\
\text { Heparin }^{*}(1 \mathrm{~mL})\end{array}$ \\
\hline Day 0 & Induction 1 & $\begin{array}{c}\text { DMEM/F12* }(489 \mathrm{~mL}) \\
\mathrm{N} 2(5 \mathrm{~mL}) \\
\text { Non-essential amino acids* }(5 \mathrm{~mL}) \\
\text { Heparin* }(1 \mathrm{~mL}) \\
\text { All-trans retinoic acid* }(0.1 \mu \mathrm{mol} / \mathrm{L})\end{array}$ \\
\hline Day 4 & Induction 2 & $\begin{array}{c}\text { DMEM/F12 }(489 \mathrm{~mL}) \\
\text { N2 }(5 \mathrm{~mL}) \\
\text { Non-essential amino acids }(5 \mathrm{~mL}) \\
\text { Heparin }{ }^{\#}(1 \mathrm{~mL}) \\
\text { All-trans retinoic acid }(0.1 \mu \mathrm{mol} / \mathrm{L}) \\
\text { Sonic hedgehog }{ }^{*}(100 \mathrm{ng} / \mathrm{mL}) \\
\end{array}$ \\
\hline Day 6 & Intermediate & $\begin{array}{c}\text { DMEM/F12 }(489 \mathrm{~mL}) \\
\text { N2 }(5 \mathrm{~mL}) \\
\text { Non-essential amino acids }(5 \mathrm{~mL}) \\
\text { Heparin }{ }^{\#}(1 \mathrm{~mL}) \\
\text { All-trans retinoic acid }(0.1 \mu \mathrm{mol} / \mathrm{L}) \\
\text { Sonic hedgehog }(100 \mathrm{ng} / \mathrm{mL}) \\
\text { cAMP }^{\sharp}(1 \mu \mathrm{mol} / \mathrm{L}) \\
\text { Ascorbic acid }^{\#}(200 \mathrm{ng} / \mathrm{mL}) \\
\end{array}$ \\
\hline Day 9 & Maturation & $\begin{array}{c}\text { DMEM/F12 }(489 \mathrm{~mL}) \\
\text { N2 }(5 \mathrm{~mL}) \\
\text { Non-essential amino acids }(5 \mathrm{~mL}) \\
\text { Heparin }{ }^{\#}(1 \mathrm{~mL}) \\
\text { BDNF, GDNF, IGF-1 } 1^{\#}(10 \mathrm{ng} / \mathrm{mL} \text { each }) \\
\text { Ascorbic acid }(200 \mathrm{ng} / \mathrm{mL}) \\
\end{array}$ \\
\hline
\end{tabular}

t-test. Three individual RT-PCR reactions were performed for each of the substrates analyzed. The oligonucleotide sequences of the primers used are listed in Table $2 \mathbf{a}, \mathbf{b}$.

Protein expression was evaluated by confocal laser scanning microscopy using FITC-conjugated secondary antibody (1:2,000, Abcam) against NESTIN (1 : 50, Abcam, ab105389), NF-H (1 : 50, Abcam, ab207176), and ChAT (1 : 50, Abcam, ab192466) primary antibodies. For the detection of TRPC1 expression, cells were stained using TRPC1 (Santa Cruz, sc-133076) primary antibody followed by Alexa Fluor 594 secondary antibody $(1: 1,000$, Thermo Fischer Scientific, A32742). Briefly, cells were fixed in $4 \%$ paraformaldehyde for $20 \mathrm{~min}$ and permeabilized using $1 \%$ Triton- $X$ in PBS for $10 \mathrm{~min}$. Cells were stained with primary antibody for $1 \mathrm{~h}$ followed by incubation for $1 \mathrm{~h}$ in either FITCconjugated or Alexa Fluor 594 secondary antibody, both at room temperature. The antibodies were diluted in a blocking buffer containing $5 \%$ goat serum, $1 \%$ BSA, and $1 \%$ Tween 20 in PBS. Cell nuclei were stained for $25 \mathrm{~min}$ at room temperature using NucBlue Live ReadyProbes Reagent (excitation/ emission 360/460 nm, Invitrogen). For cells stained with TRPC1, actin filaments were counterstained for $1 \mathrm{~h}$ with Alexa Fluor 488 Phalloidin (1 : 1,000,
Thermo Fischer Scientific). Cells were mounted using ProLong ${ }^{\mathrm{TM}}$ Gold Antifade Mountant (Thermo Fischer Scientific) and imaged using the Olympus FluoView FV1000 (Olympus) laser scanning confocal microscope.

\section{PEMF exposure system}

The PEMF system used has been previously described (Crocetti et al., 2013; Parate et al., 2017; Yap et al., 2019). Briefly, the PEMF exposure system produces spatially homogeneous, time-varying magnetic fields, consisting of barrages of $20 \times 150 \mu \mathrm{s}$ on and off pulses for $6 \mathrm{~ms}$ repeated at a frequency of $15 \mathrm{~Hz}$. hDPSC cultures were exposed once for $10 \mathrm{~min}$ at PEMF amplitudes ranging between 1 and $3 \mathrm{mT}$. Control samples $(0 \mathrm{mT})$ were manipulated exactly as experimental samples, including placement into the PEMF-generating apparatus for the designated time, except that the apparatus was not set to generate magnetic fields.

\section{Calcium flux and choline release assay}

Cholinergic calcium mobilization was measured using the Calcium Flux Assay Kit (Abcam, ab233472) in response to different concentrations of carbachol. The percentage increase was calculated relative to control cultures in basal growth medium on either 
Table 2a. Primer sequences.

\begin{tabular}{|c|c|c|}
\hline Gene name & Primer & Sequence \\
\hline \multirow{2}{*}{ NESTIN } & Forward & 5'-CTGCTACCCTTGAGACACCTG-3' \\
\hline & Reverse & 5'-GGGCTCTGATCTCTGCATCTAC-3' \\
\hline \multirow{2}{*}{ ChT } & Forward & 5'-AGGAGGGTATATCAATGGCACA-3' \\
\hline & Reverse & 5'-CCTTTGAACGCATAGGTTTTGC-3' \\
\hline \multirow{2}{*}{ ISL 1} & Forward & 5'-TACGGGATCAAATGCGCCAA-3' \\
\hline & Reverse & 5'-CACACAGCGGAAACACTCGAT-3' \\
\hline \multirow{2}{*}{ ISL 2} & Forward & 5'-CTGCAAGCGGGACTACGTC-3' \\
\hline & Reverse & 5'-CACTCGATGTGGTACACGC-3' \\
\hline \multirow{2}{*}{ ENO-2 } & Forward & 5'-TCGCTTTGCCGGACATAACT-3' \\
\hline & Reverse & 5'-GACACATCGTTCCCCCAAGT-3' \\
\hline \multirow{2}{*}{ OLIGO 2} & Forward & 5'-TGGCTTCAAGTCATCCTCGTC-3' \\
\hline & Reverse & 5'-ATGGCGATGTTGAGGTCGTG-3' \\
\hline \multirow{2}{*}{$H B-9$} & Forward & 5'-GATGCCCGACTTCAACTCCC-3' \\
\hline & Reverse & 5'-GCCGCGACAGGTACTTGTT-3' \\
\hline \multirow{2}{*}{ TUB-3 } & Forward & 5'-CAGCAAGGTGCGTGAGGAG-3' \\
\hline & Reverse & 5'-TGCGGAAGCAGATGTCGTAG-3' \\
\hline \multirow{2}{*}{$N F-H$} & Forward & 5'-GCCAAGGTGGAGGTGAAGGA-3' \\
\hline & Reverse & 5'-TGGTCTGTGCTGGAGGATTTTT-3' \\
\hline \multirow{2}{*}{$A C h$} & Forward & 5-GGGTGGTAGACGCTACAACC-3' \\
\hline & Reverse & 5'-GTGCCCTCAAAACCTGGGTAT-3' \\
\hline \multirow{2}{*}{$N F-M$} & Forward & 5'-GAGCGCAAAGACTACCTGAAGA-3' \\
\hline & Reverse & 5'-CAGCGATTTCTATATCCAGAGCC-3' \\
\hline \multirow{2}{*}{ SMN } & Forward & 5'-ATGAGCTGTGAGAAGGGTGTTG-3' \\
\hline & Reverse & 5'-TTGCCACATACGCCTCACATAC-3' \\
\hline \multirow{2}{*}{ ChAT } & Forward & 5'-CAGCCCTGCCGTGATCTTT-3' \\
\hline & Reverse & 5'-TGTAGCTGAGTACACCAGAGATG-3' \\
\hline \multirow{2}{*}{ VAChT } & Forward & 5'-TGGCGCTGTTACTGGACAAC-3' \\
\hline & Reverse & 5'-TTCGCTCTCCGTAGGGTAGC-3' \\
\hline \multirow{2}{*}{ PCNA } & Forward & 5'-CCTGCTGGGATATTAGCTCCA-3' \\
\hline & Reverse & 5'-CAGCGGTAGGTGTCGAAGC-3' \\
\hline \multirow{2}{*}{ TRPC1 } & Forward & 5'-AGGAACTAGCCCGGCAATGTA-3' \\
\hline & Reverse & 5'-GCTCGTCACTAGACGTATGGTTT-3' \\
\hline \multirow{2}{*}{ TRPC6 } & Forward & 5'-GTTATGTTCGGATTGTGGAAGCA-3' \\
\hline & Reverse & 5'-ACATCATGGGAGAACCGTGTC-3' \\
\hline \multirow{2}{*}{ ChAT } & Forward & 5'-CAGCCCTGCCGTGATCTTT-3' \\
\hline & Reverse & $\begin{array}{l}5^{\prime} \text {-TGTAGCTGAGTACACCAGAGATG-3' } \\
\end{array}$ \\
\hline \multirow{2}{*}{$H B-9$} & Forward & 5'-GATGCCCGACTTCAACTCCC-3' \\
\hline & Reverse & 5'-GCCGCGACAGGTACTTGTT-3' \\
\hline \multirow{2}{*}{ PCNA } & Forward & 5'-CCTGCTGGGATATTAGCTCCA-3' \\
\hline & Reverse & 5'-CAGCGGTAGGTGTCGAAGC-3' \\
\hline \multirow{2}{*}{ TRPC1 } & Forward & 5'-AGGAACTAGCCCGGCAATGTA-3' \\
\hline & Reverse & 5'-GCTCGTCACTAGACGTATGGTTT-3' \\
\hline \multirow{2}{*}{ TRPC6 } & Forward & 5'-GTTATGTTCGGATTGTGGAAGCA-3' \\
\hline & Reverse & 5'-ACATCATGGGAGAACCGTGTC-3' \\
\hline \multirow{2}{*}{ TRPM7 } & Forward & 5'-CAGGCTCTATGATCGCAGGAG-3' \\
\hline & Reverse & 5'-TTTGAACTCGTTGTCTGTGAGG-3' \\
\hline & Forward & 5'-GACTTCCAGAGTACCTGAGCA-3' \\
\hline TRPM8 & Reverse & 5'-GCAGGCATTGACTCCGTCC-3' \\
\hline
\end{tabular}


Table 2a. Primer sequences.

\begin{tabular}{|c|c|c|}
\hline \multirow{2}{*}{ Gene name } & Primer & Sequence \\
\hline \multirow{2}{*}{ TRPV1 } & Forward & 5'-ACTGGAGGAGTAAACACAGGT-3' \\
\cline { 2 - 3 } & Reverse & 5'-TGGAGCTATTCCGATAGTGCAA-3' \\
\hline \multirow{2}{*}{$T R P V 2$} & Forward & $5^{\prime}$-GCAAGTGTGGCTATGCCCA-3' \\
\cline { 2 - 3 } & Reverse & 5'-CCAAAGGCGTCGGTAGGAA-3' \\
\hline \multirow{2}{*}{ CACNA2D2 } & Forward & 5'-ATGGAAAACCGCAGACAAGAC-3' \\
\cline { 2 - 3 } & Reverse & 5'-CCGGGTAGTAGCGAGTGAC-3' \\
\hline \multirow{2}{*}{ CACNA1C } & Forward & 5'-AATCGCCTATGGACTCCTCTT-3' \\
\cline { 2 - 3 } & Reverse & 5'-GCGCCTTCACATCAAATCCG-3' \\
\hline \multirow{2}{*}{ GAPDH } & Forward & 5'-GTGGACCTGACCTGCCGTCT-3' \\
\cline { 2 - 3 } & Reverse & 5'-GGAGGAGTGGGTGTCGCTGT-3' \\
\hline
\end{tabular}

substrate and performed in triplicates. The production of acetylcholine was assessed by quantifying the concentration of choline present in the bathing medium. hDPSC were differentiated on 2DG or glass for $15 \mathrm{~d}$ (Table 1) and, then, exposed to PEMFs $(2 \mathrm{mT})$ or not $(0 \mathrm{mT})$. After $24 \mathrm{~h}, 50 \mu \mathrm{L}$ of the medium was collected and used to quantify total choline (colorimetric/fluorometric Choline/Acetylcholine Assay Kit, Abcam ab65345) at an excitation/emission ratio of 535/587 $\mathrm{nm}$ using a fluorometric plate reader (Infinite m200, Tecan Trading AG, Männedorf, Switzerland). The total choline was calculated as per manufacturer instructions $(\mu \mathrm{mol} / \mathrm{L} / \mu \mathrm{L})$ and normalized against $0 \mathrm{mT}$.

\section{Absolute quantification of cation channel expression}

Absolute gene expression of TRP channels of cells on both glass and 2DG substrates with and without neurogenic induction was ascertained by RT-PCR. Using the generated CT values, the copy number of the standards and the experimental groups was calculated (Madhvi et al., 2017). Briefly, hDPSC were seeded onto 2DG or glass and RNA collected (Purelink RNA Mini Kit, Invitrogen) 1 (D1) and 4 (D4) d post neurogenic induction. For the creation of standard curves, RNA was harvested from hDPSCs grown in flasks, followed by reverse transcription using iScript RT Supermix (Bio-Rad). To establish the amplification efficiency $\left(E_{A M P}\right)$, a conventional PCR, using the same set of primers (Table 2a, b) and ITaqMan ${ }^{\circledR}$ system (Bio-Rad), was performed on $25 \mathrm{ng}$ of cDNA obtained from the RNA isolated from hDPSC grown in a flask and amplified for 30 cycles (Bio-Rad Thermal cycler) to generate the desired PCR product. Then, the PCR product was purified using a gel extraction kit (PureLinkTM Quick Gel Extraction Kit, Invitrogen) and quantified using a NanoDrop (Thermo Fisher Scientific). Copy number from the purified DNA was calculated using the predicted PCR product size and a 650 molar mass per base pair ( $\mathrm{g} / \mathrm{mol} / \mathrm{bp})$. Using the copy number calculated, the DNA was serially diluted in 10-fold dilutions from 100 to $10^{-6} \mathrm{ng} / \mu \mathrm{L}$ concentration. Standard curves were created, and the slope and intercept were calculated.
$E_{A M P}$ was ascertained using the slope as indicated in Equation 1. Along with the standards, the samples were also run on the same plate. Using Equation 2, the absolute copy number from the samples (per ng) was determined by dividing the copy number amount by 25 , as $25 \mathrm{ng}$ of cDNA were used for the RT-PCR reaction.

$$
\begin{array}{ll}
E_{A M P}=10^{(-1 / \text { slope })} & \text { Equation 1 } \\
X 0=E_{A M P}^{(b-C T)} & \text { Equation 2 }
\end{array}
$$

where $X 0$ is the initial number of target copies, $E_{A M P}$ is the amplification efficiency, $b$ is the $y$-intercept of the standard plot, and CT is the cycle threshold value (Madhvi et al., 2017).

\section{Antibiotic treatment}

Aminoglycoside antibiotics preclude the developmental effects of PEMFs because of their ability to antagonize TRPC1 channels (Yap et al., 2019). PS (Invitrogen) was used to confirm the involvement of TRP channels in the neurogenic responses rendered by PEMF, as previously shown (Yap et al., 2019). $15 \times 10^{3}$ cells were seeded onto either $2 \mathrm{DG}$ or glass in $500 \mu \mathrm{L} /$ well neurogenic induction medium 1 (Table 1). $24 \mathrm{~h}$ later, $250 \mu \mathrm{L}$ of medium was removed from each well and PS was added at $2 \times$ concentration. After gentle mixing, $250 \mu \mathrm{L}$ medium was added back to each well bringing the final volume to $500 \mu \mathrm{L}$ and PS to a final concentration of $1 \%$. Following PS incubation for $2 \mathrm{~h}$, cells were either exposed to PEMFs ( $2 \mathrm{mT})$ or not $(0 \mathrm{mT})$, before replacement with fresh medium $24 \mathrm{~h}$ later. Cells were allowed to grow for another additional $3 \mathrm{~d}$, after which neurogenic induction medium 1 was changed to neurogenic induction medium 2 (Table 1). After 24 h, RNA was extracted and gene expression analyzed by RT-PCR. Timeline-matched cells on glass without PS or PEMF $(0 \mathrm{mT})$ exposures were used as controls.

\section{Cell proliferation}

Cell proliferation was assessed using the colorimetric MTS assay. 7,000 cells were seeded onto glass or 2DG in growth medium and cell proliferation was assessed after day 1,3 , and 5 using the MTS reagent (CellTiter 
96 AQueous One Solution Assay, Promega). Briefly, $60 \mathrm{~min}$ after incubation with MTS reagent, absorbance at $490 \mathrm{~nm}$ was measured using a plate reader (Infinite $\mathrm{m} 200)$. Then, the viability of the adherent cells was determined by MTS reduction. Negative controls were substrates devoid of cells.

For the assessment of proliferation using genetic markers, 7,000 cells (low cell-density) or 30,000 cells (high cell-density) were seeded onto glass or 2DG and neurogenic induction commenced with basal growth medium, as described under "Neuronal differentiation". The expression of PCNA, NF-M, and HB-9 at day 5 was analyzed by RT-PCR.

\section{ROS and superoxide quantification}

ROS and superoxide production were quantified using a cellular ROS/Superoxide Detection Assay Kit (cell-based) (Abcam, ab139476). hDPSCs were seeded onto either 2DG or glass and neurogenic differentiation was induced using neurogenic induction medium 1 . After $24 \mathrm{~h}$ of induction, $1: 500$ oxidative stress detection reagent (fluorescein, green) and $1: 2,500$ superoxide detection reagent (rhodamine, orange) were thoroughly mixed in fresh culture medium and thereafter used to replace the culture medium of recipient cell wells or applied to empty wells to be used as blanks. Positive and negative controls provided in the kit were used to validate the assay. DPSCs were exposed to PEMFs at 0,2 or $3 \mathrm{mT}$ for $10 \mathrm{~min}$ and returned to a standard tissue culture incubator for $1 \mathrm{~h}$. Then, plates were read using a fluorescence plate reader (Infinite m200, Tecan Trading AG, Männedorf, Switzerland) at excitation/ emission 488/520 nm for fluorescein and 550/610 nm for rhodamine. The fluorescent signal from the experimental groups was subtracted from that of the blanks and ROS and superoxide levels were normalized and expressed as fold change against unstimulated DPSCs $(0 \mathrm{mT})$ on glass.

\section{Statistical analysis}

All experiments were performed in independent biological triplicates, with results presented as mean \pm standard deviation of the experimental repeats unless otherwise stated in the figure legend. Data were analyzed using Lavene's tests for equality of variances and Shapiro-Wilk test for normality. To compare the mean difference between the control and experimental groups and among the experimental groups, the statistical significance was analyzed by one-way or two-way ANOVA and post-hoc Bonferroni test with a global significance level set at $5 \%$. For the mean assessment of two experimental groups, a twosample Student's $t$-test was performed.

\section{Results}

\section{Neurogenic differentiation on 2DG}

The potential of 2DG to promote the differentiation of hDPSCs into the neurogenic lineage was first investigated. To this end, hDPSCs were seeded onto uncoated glass (control) or graphene-coated glass substrates of very high purity and negligible structural defects, as reflected by the surface mapping (Fig. 1a). Next, hDPSCs were differentiated and the expressions of key neuron genes and proteins ascertained to monitor neurogenic progression on either substrate. hDPSCs on 2DG exhibited higher expression levels of genes associated with later neural (NF-M, NF-H) and neurogenic-related (SMN, $V A C h T, C h A T)$ in vitro differentiation (Fig. 1b; 2DG/ glass ratio $>1$, dashed line), whereas early (NESTIN) and transitional neurogenic (OLIGO-2, ISL-1, ISL$2, E N-1, C h T)$ genes were more expressed on glass (Fig. 1b; 2DG/glass ratio < 1), suggesting accelerated neurogenic progression on 2DG (Madanagopal et al., 2020). This same trend was also corroborated at the protein level (Fig. 1c,f), as observed for ChAT, which is responsible for neurotransmitter production. Accordingly, hDPSCs differentiated on 2DG exhibited more carbachol-induced calcium uptake (Fig. 1d), whereby the apparent desensitization of the response corroborates its cholinergic origin implicated in signal processing (Asrican et al., 2016). As the progression of differentiation is influenced by cell density reaching a critical threshold for optimal paracrine factor release as well as for providing critical levels of cellcontact-mediated signaling (Peccoud et al., 2009), an acceleration of proliferation would produce a similar trend in gene expression. Accordingly, the expression of TRPC1, a Ca ${ }^{2+}$-permeable cation channel supporting neuronal proliferation (Abramowitz and Birnbaumer, 2009), was upregulated in hDPSCs on 2DG (Fig. 1e,f).

Given the accepted importance of calcium entry during stem cell-derived neurogenesis (Toth et al., 2016), cation-permeable channel expression was monitored during early neurogenic induction on 2DG and glass. The expression levels of TRPC1, TRPC6, TRPV1, and TRPM 8 were the highest in hDPSCs grown on 2DG (blue) $1 \mathrm{~d}$ after neurogenic induction (day 1, Fig. 2) and reverted to control levels by day 4. hDPSCs grown on glass (red) showed attenuated versions of this same trend, with the notable exception of TRPV1, which instead decreased from the date of plating on glass. Among TRP channels, the expression levels were the highest for TRPC1 $\left(10^{8}\right.$ copies/ng cDNA), followed by TRPC6 ( $10^{7}$ copies/ng cDNA) and TRPM8 (106 copies/ng cDNA) and were overall higher on 2DG at day 1, whereas TRPV1 expression ( $10^{7}$ copies/ng cDNA) was reduced at day 1 on glass. Hence, 2DG synchronized and enhanced the developmental modulation of this set of TRP channels. By contrast, the voltage-gated calcium channels $C A C N A 2 D 2$ and $C A C N A 1 C$ displayed quite disparate developmental expression profiles from these TRP channel family members. In summary, TRPC1 was the most highly expressed channel examined on the first day following neurogenic induction, particularly for hDPSCs grown on 2DG. Hence, the first day after neurogenic induction 


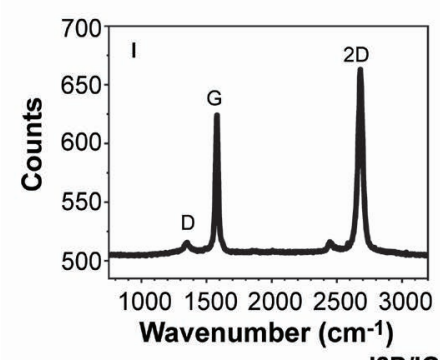



ID/IG

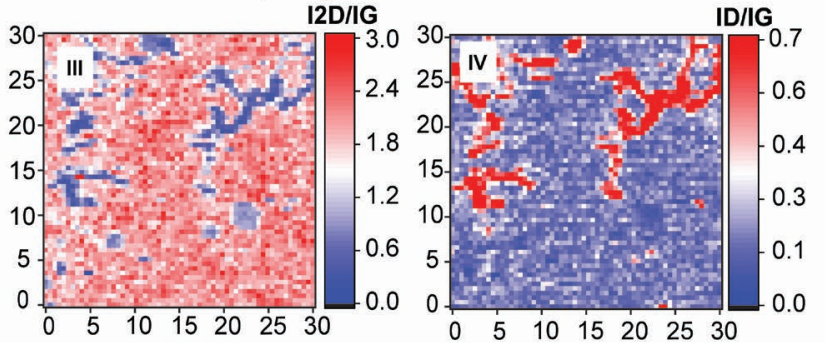

C
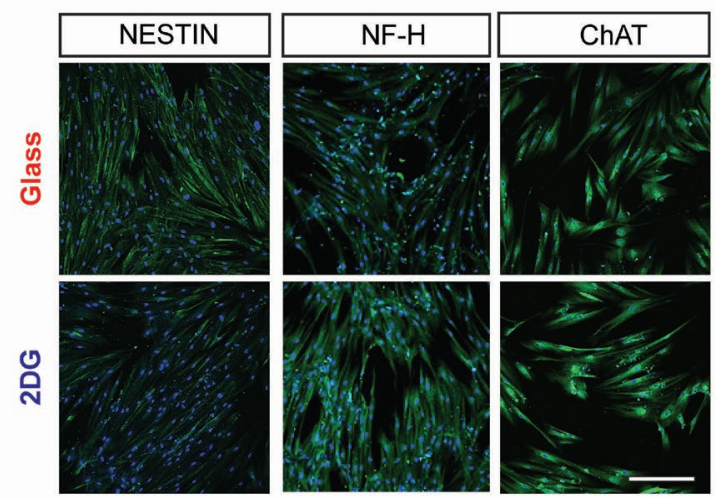

e
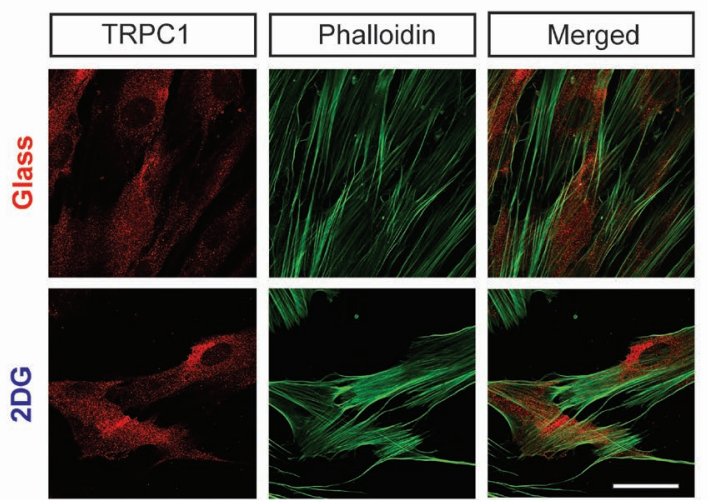

b

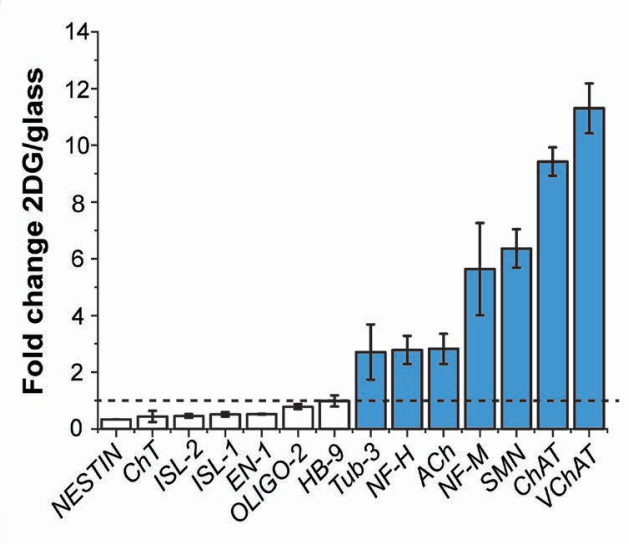

d

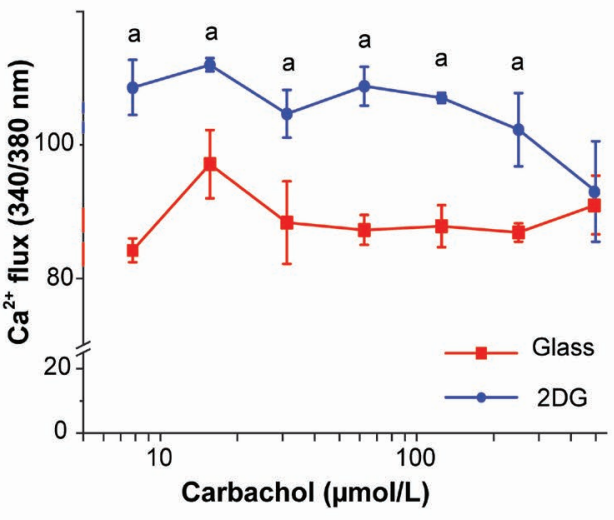

f
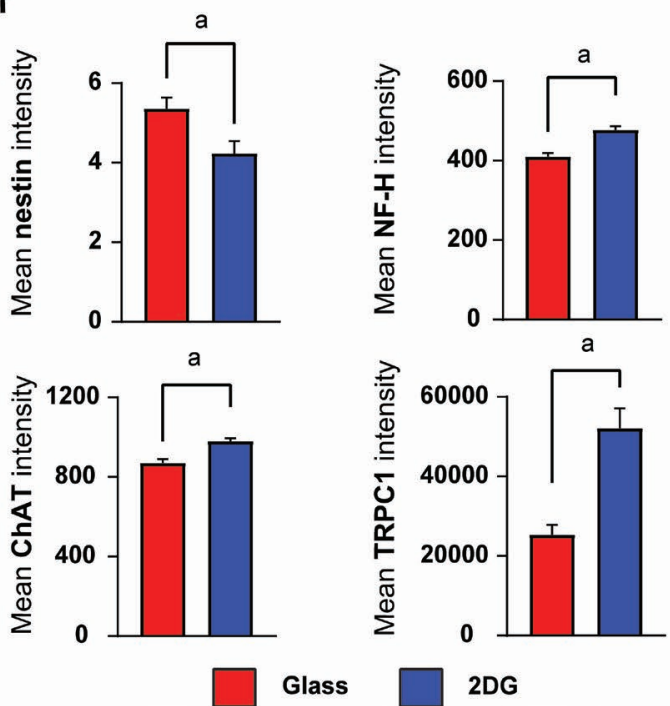

Fig. 1. 2DG accelerated the expression of neurogenic genes and proteins in hDPSCs. (a) 2DG characterization. I: Raman spectrum obtained from 2DG presented the typical peak signatures. II and III: the analyses of FWHM 2D $\left(\mathrm{cm}^{-1}\right)$ and I2D/IG ratio (2D band over $\mathrm{G}$ band intensity) mappings indicated the successful transfer of a continuous layer of graphene onto the glass surface. IV: the ID/IG ratio (D band over $\mathrm{G}$ band intensity) demonstrated negligible defects on the graphene coating. (b,c) Cells on 2DG showed significant increases in the expression of $(\mathbf{b})$ neurogenic genes and (c) proteins associated with advanced neurogenic induction (blue bars) compared to glass. A student's $t$-test analysis was performed on 2DG vs. glass with blue bars indicating a statistical difference of $p<0.05$. Dashed line denotes the ratio level equal to 1. Scale bar: $400 \mu \mathrm{m}$. (d) Carbachol-induced calcium influx was more for hDPSCs induced to differentiate on 2DG (percentage increase from plated hDPSCs on glass and in basal growth medium). (e) TRPC1 expression was upregulated on 2DG relative to glass (left, red) and was not associated with signs of overt stress, as indicated by phalloidin staining (middle, green). Scale bar: $40 \mu \mathrm{m}$. (f) Quantification of fluorescent protein signal intensity (expressed as arbitrary fluorescence unit after background normalization) for the indicated neurogenic markers. Statistical analysis was performed using Student's $t$-test on the mean of at least 100 cells, with error bars expressed as the standard error of the mean. ${ }^{a}$ indicates statistical differences between 2DG (blue) and glass (red) with $p<0.05$. 


\section{Transcript copy number per ng cDNA $\square$ Glass $\square$ 2DG}
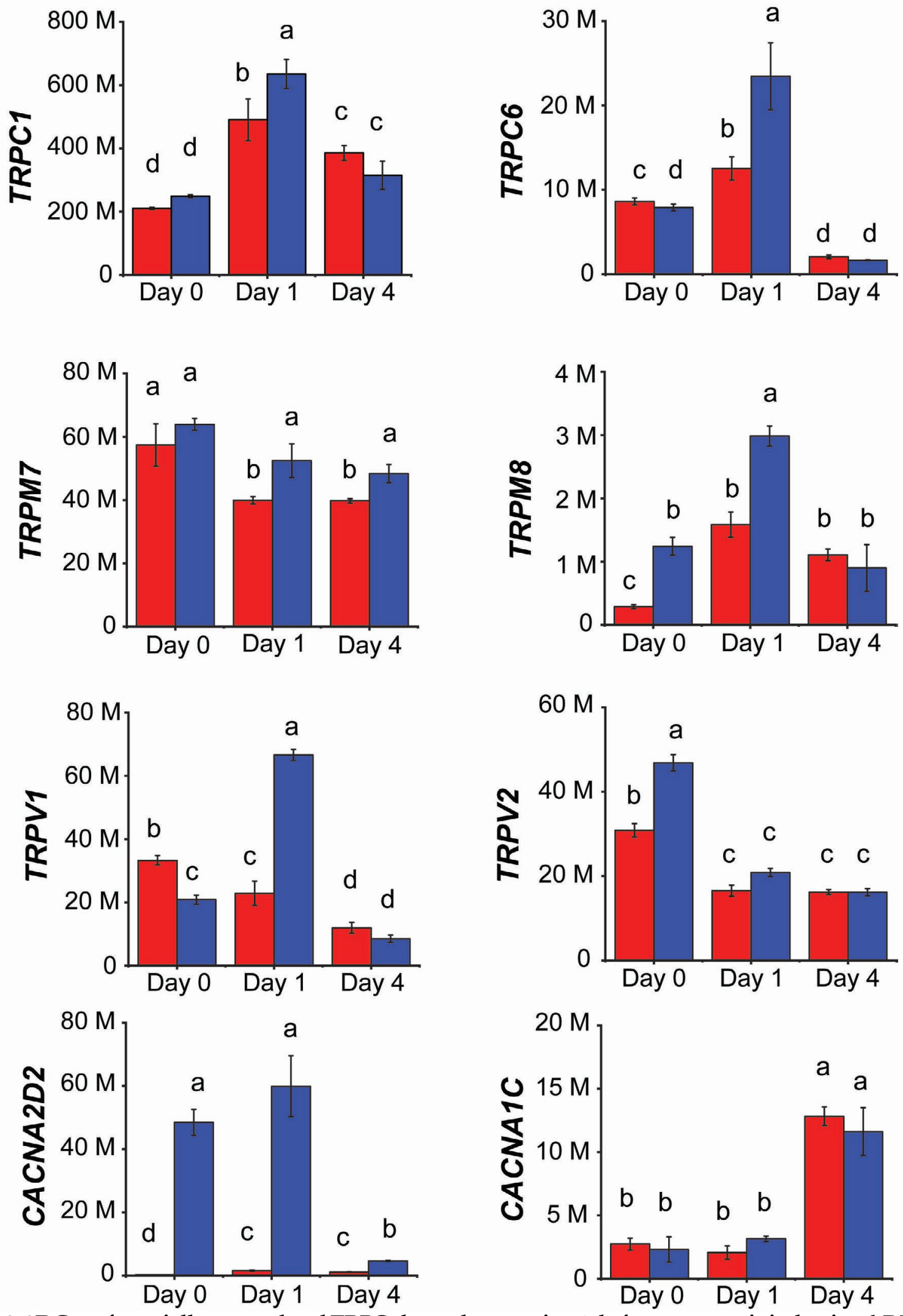

Fig. 2. 2DG preferentially upregulated TRPC channel expression $1 \mathrm{~d}$ after neurogenic induction. hDPSCs were seeded on to 2DG or glass followed by neurogenic induction. The absolute expression of selected TRP or voltage-gated calcium channels, as indicated, were quantified and represented as transcript copy number/ng of cDNA. $M$ = millions; data from each panel were analyzed using two-way ANOVA and Tukey's honest significant difference tests to evaluate the differences in expressions calculated for glass and 2DG at the different time points. Bars amarked with identical letters $\left(a,{ }^{a}\right)$ are statistically similar, whereas bars marked with different letters $(\stackrel{a}{a}$,$) are statistically different by p<0.05$. 
provided the best window of opportunity to exploit the known neurogenic attributes of TRP channels, in particular TRPC1 (Toth et al., 2016), an attribute conferred by plating hDPSCs onto 2DG.

TRPC1 is necessary and sufficient to confer developmental sensitivity to brief exposure PEMFs (Kurth et al., 2020; Yap et al., 2019). Therefore, whether PEMF exposure could enhance neurogenesis was tested by targeting the developmental expression of TRPC1 conferred by 2DG. hDPSCs were seeded onto 2DG or glass and exposed for $10 \mathrm{~min}$ to PEMFs of amplitudes ranging between 1 and $3 \mathrm{mT}$ on the first day following neurogenic induction (day 1). Transcriptional analyses were conducted on day 1 (day 2), 4 (day 5), and 7 (day 8) following neurogenic induction (day 1). hDPSCs plated onto 2DG exhibited robust upregulations of PCNA, NF-M, and HB-9 at day 5 in response to $2 \mathrm{mT}$ PEMF exposure (Fig. 3, top) at day 1, when TRPC1 was most highly expressed (Fig. 2). In contrast, hDPSCs plated onto glass (Fig. 3 , bottom) did not show a consistent response trend. hDPSCs plated onto glass generally showed the best response to stronger PEMF exposures of $3 \mathrm{mT}$, although the overall effect sizes were much smaller than those observed for hDPSCs plated on 2DG, often failing to surpass control levels $(0 \mathrm{mT} ; 1.0)$. Hence, hDPSCs plated onto glass required stronger PEMF exposure ( $3 \mathrm{mT})$ to achieve smaller neurogenic effects.
Cell density is a determinant factor in responsiveness to PEMFs. TRPC1 expression and, consequently, developmental response to PEMFs mirror early cell expansion prior to reaching full cell confluency (Yap et al., 2019). Accordingly, seeding hDPSCs at higher densities that restricted cell expansion, precluded proliferative response to PEMF exposure (Fig. 4a), reinforcing the criteria for early PEMF exposure (Fig. 2). PEMF exposure strongly increased the release of choline, the enzymatic breakdown product of acetylcholine neurotransmitter, into the bathing medium of hDPSC cultures differentiated on 2DG, whereas hDPSC s differentiated on glass failed to demonstrate PEMF-induced choline release (Fig. 4b vs. Fig. 1d), reinforcing that neurogenic induction was stronger on 2DG. PEMFs activate a TRPC1-mitochondrial axis promoting in vitro proliferation and subsequent differentiation (Kurth et al., 2020; Yap et al., 2019). Thus, the ability of 2DG in combination with PEMF exposure to augment the production of ROS and superoxide, products of cellular respiration, was investigated. 2DG alone was sufficient to raise ROS levels over glass (Fig. 4c), whereas DPSCs on either 2DG or glass exhibited the highest levels of ROS (Fig. 4c) and superoxide (Fig. 4d) following exposure at $2 \mathrm{mT}$, which receded at $3 \mathrm{mT}$, reinforcing developmental specificity to the $2 \mathrm{mT}$ peak.
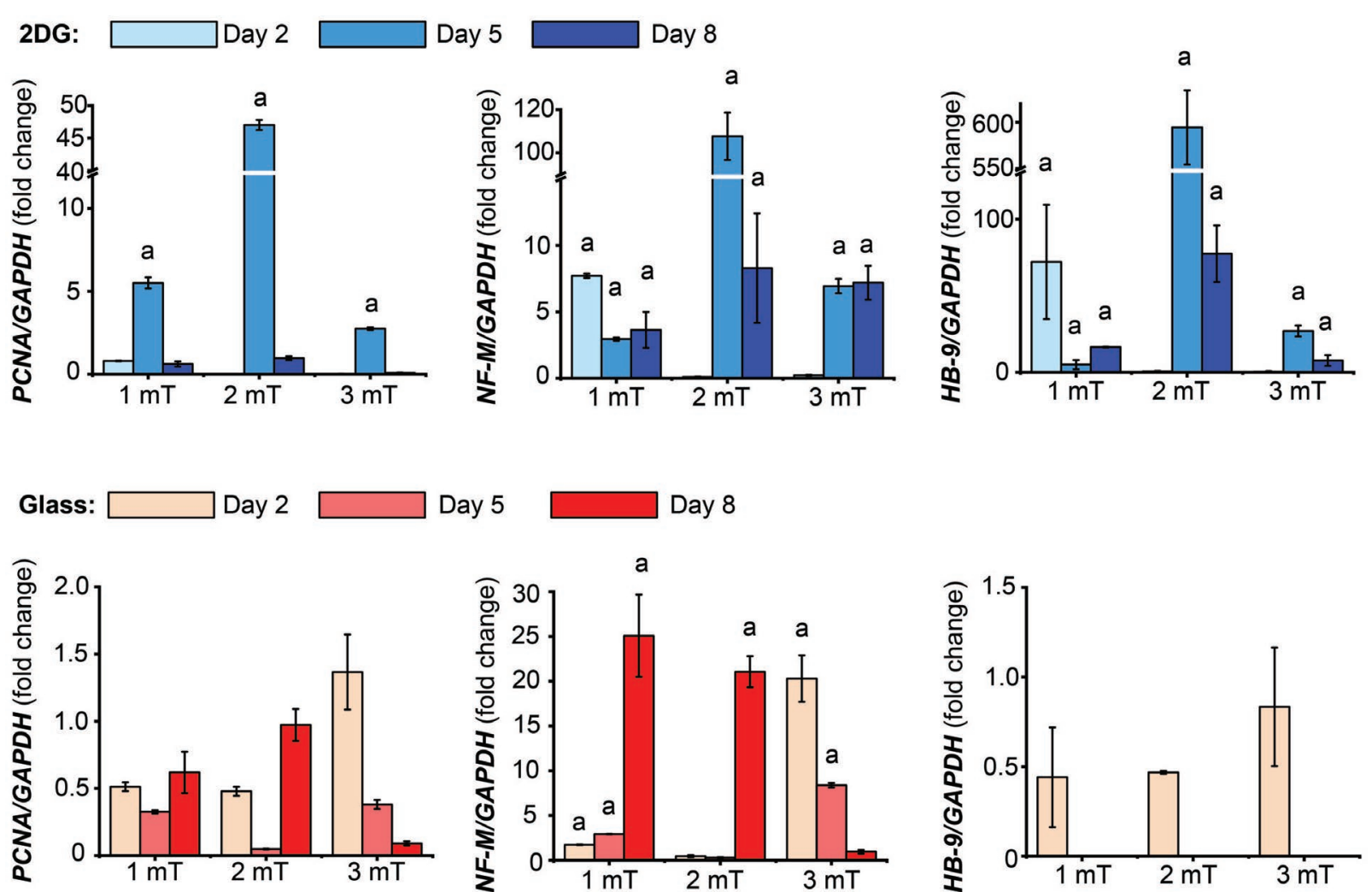

Day 5

Day 8
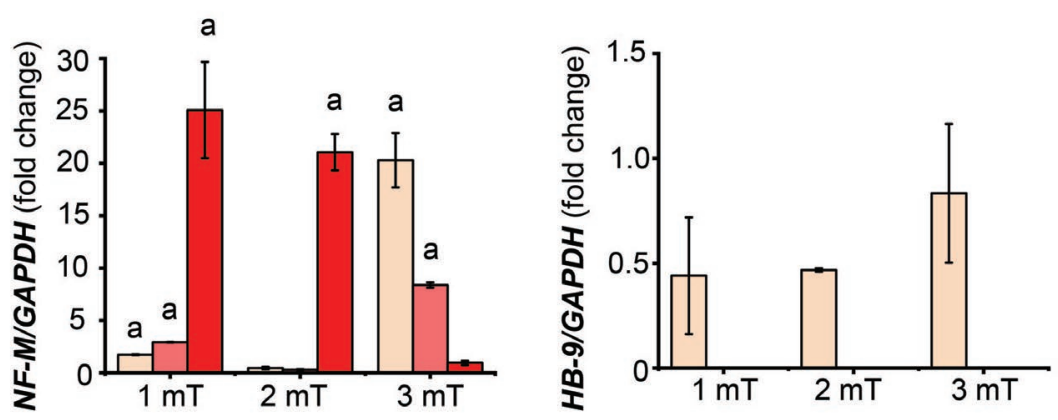

Fig. 3. Neurogenic gene expression was further amplified on 2DG in response to PEMF exposure. 2DG (top, shades of blue) enhanced the expression levels of PCNA, NF-M, and HB-9 as compared to glass (bottom, shades of red) following a single 10 min exposure to PEMFs at $2 \mathrm{mT}$. The synergistic effects of $2 \mathrm{DG}$ and PEMF exposure were most pronounced at day 5, whereas the expressions of all genes at day 8 were similar or lower than those observed for non-stimulated $(0 \mathrm{mT})$ cells. One-way ANOVA analysis was used to compare the expression of each PEMF amplitude against its respective $0 \mathrm{mT}$ on the same day. ${ }^{\mathrm{a}} p<0.05$ compared to respective $0 \mathrm{mT}$ scenario (no fold change $=1$ ). 
Aminoglycoside antibiotics antagonize TRPC1mediated calcium entry (Matsumura et al., 2011) and, hence, preclude response to PEMF exposure (Yap et al., 2019). Indeed, the commercial formulation of PS, conventionally used in tissue culturing paradigms, could recapitulate the attenuation of cell differentiation observed with the administration of the pure aminoglycoside antibiotics (Yap et al., 2019). Accordingly, the growth of hDPSCs in PS depressed cell proliferation on both glass and 2DG (Fig. 4e). Moreover, the application of PS, timed to coincide with PEMF exposure, was sufficient to attenuate the PEMF-induced upregulation of PCNA observed in hDPSCs plated on 2DG (7\% with PS; Fig. $4 \mathbf{f}$ ) and glass (16\% with PS). Hence, the use of PS should be avoided when aiming to exploit the neurogenic attributes of either graphene or PEMF treatment.
The sum of the presented results corroborated that TRPC1 is required for the developmental efficacy of this specific magnetic field paradigm applied to hDPSCs - as previously reported in skeletal myoblasts (Yap et al., 2019), MSCs (Parate et al., 2017; 2020), and reconstituted cell systems (Kurth et al., 2020) - which, moreover, could be enhanced with the implementation of graphene substrates.

\section{Discussion}

Cell-based tissue engineering holds great potential for regenerative endodontics. However, despite recent breakthroughs in this field, the engineering of a dental pulp with nociceptive properties remains a major challenge. The absence of innervation a

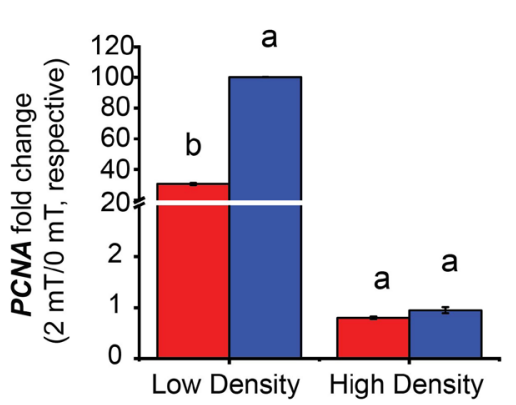

d

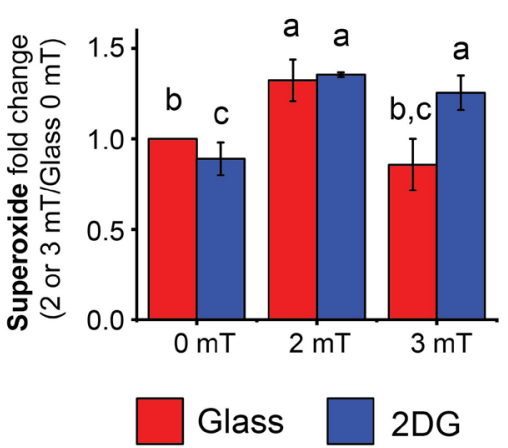

b



e

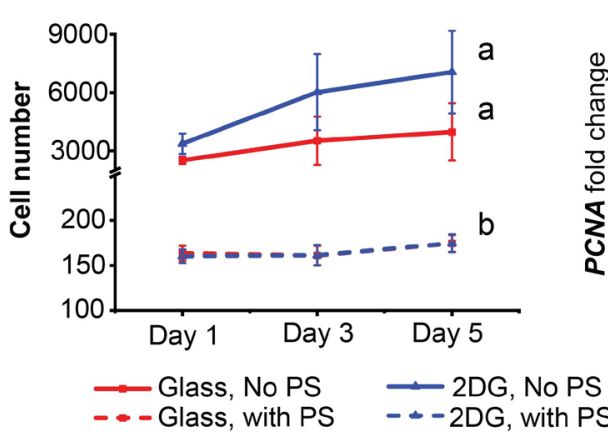

C

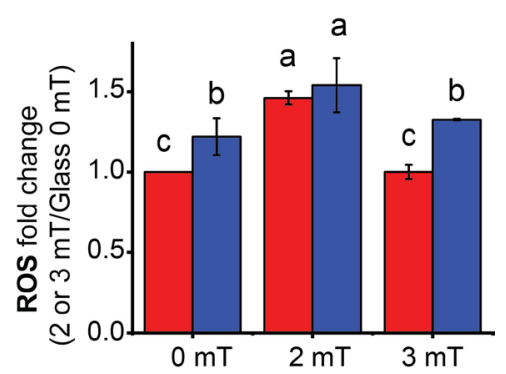

f

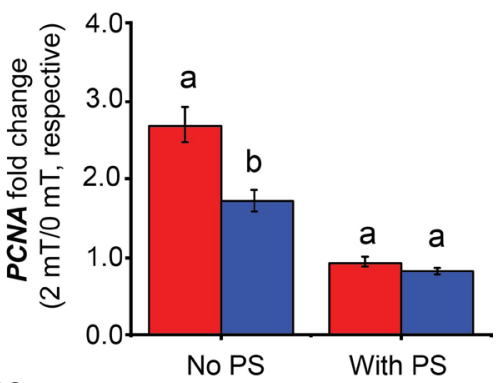

Fig. 4. Interactions between proliferation and differentiation statuses and response to PEMFs/2DG. (a) Plating hDPSCs at high density precluded PEMF-induced proliferation enhancement on 2DG. Total RNA was isolated at day 1 (Fig. 2). (b) PEMF-induced choline release was significantly enhanced from hDPSCs differentiated for $15 \mathrm{~d}$ on 2DG (blue), compared to glass (red). (c) ROS and (d) superoxide production upon PEMF exposure at $2 \mathrm{mT}$ and $3 \mathrm{mT}$ on both glass (red) and 2DG (blue). ROS and superoxide levels were normalized against those of unexposed hDPSCs $(0 \mathrm{mT})$ on glass. (e) Time course of hDPSC proliferation on 2DG (blue) or glass (red) with (dashed line) or without PS (solid line). (f) The presence of PS during PEMF exposure attenuated the typical PEMF-induced upregulation of PCNA expression on both glass (red) or 2DG (blue). In this instance, PCNA expression was overall lower on 2DG due to its downregulation at higher cell density compared to glass at the time of RNA collection. hDPSC cultures with or without PS were exposed to PEMFs (2 mT) and gene expression was analyzed using RT-PCR at day 5. Data are presented relative to respective unexposed $(0 \mathrm{mT})$ cultures on glass. $(\mathbf{a}, \mathbf{c}, \mathbf{d}, \mathbf{f})$ Data are shown after normalization to $0 \mathrm{mT}(2 \mathrm{mT}$ or $3 \mathrm{mT} / 0 \mathrm{mT})$ where no fold change $=1$. $(\mathbf{a}, \mathbf{f})$ Data were analyzed using one-way ANOVA whereby 2DG was compared to glass for each condition tested (e.g. density: low vs. low, high vs. high, etc.). (b) Data were analyzed using a paired $t$-test with error bars $=5 \%$. (c-e) Two-way ANOVA was used to analyze data (e.g., glass vs. 2DG and different intensities). (e) Cell number was calculated using a standard curve for absorbance $v$ s. cell number. In all panels, different letters denote statistical difference with $p<0.05$. 
represents a major drawback as it is essential for nociception, angiogenesis as well as functional regeneration of the pulp itself (Byers et al., 2003; Xuan et al., 2018). Although several biomaterials have been found to promote the neurogenic differentiation of stem cells, inherent limitations such as toxicity and partial differentiation have hampered their potential for clinical exploitation (Bahrami et al., 2017; EbrahimiBarough et al., 2015; O'Brien, 2011; Rosa et al., 2012). Independently, both graphene substrates (Guo et al., 2016; Kim et al., 2015; Luong-Van et al., 2020) and PEMFs (Ma et al., 2016) have been demonstrated to support neurogenic differentiation. Nonetheless, further optimization was required before potential clinical implementation. The present study showed that the seeding of hDPSCs onto graphene followed by a single PEMF exposure (10 min) $24 \mathrm{~h}$ later created a more conducive environment for their subsequent neurogenic differentiation. These initial results will serve to foster the development of graphene-based and PEMF-assisted strategies for the tissue engineering of a functional dental pulp with nociceptive capacities.

Graphene films grown by chemical vapor deposition (2DG) are highly pure and are readily deposited onto most two- or three-dimensional surfaces (Morin et al., 2017). 2DG supports cell attachment and proliferation (Kalbacova et al., 2010; Lee et al., 2011; Li et al., 2015; Xie et al., 2019; Xie et al., 2015) and has emerged as a promising and affordable platform to induce neurogenic differentiation with functional signaling capacity (Luong-Van et al., 2020). 2DG activates FAK and MAPK cascades (Li et al., 2015; Li et al., 2012; Xie et al., 2019) as well as upregulates the expression of F-actin, vinculin, BMP2, and MYH (Dubey et al., 2020; Li et al., 2015; Xie et al., 2019; Xie et al., 2015), implicating cellular mechanotransduction as part of its mode of action (Engler et al., 2006; Luong-Van et al., 2020; Wang et al., 2012). The present study showed that TRPC1, previously implicated in cellular mechanotransduction, is preferentially upregulated by 2DG (Clapham, 2003; Crocetti et al. 2014). TRPC1 contributes also to the calcium entry that is required for calcineurin/NFAT transcriptional regulation of neurogenesis (Toth et al., 2016). Accordingly, PEMF stimulation recruits a calcineurin/NFAT transcriptional cascade common to cellular mechanotransduction and impinges upon TRPC1, without vicariously producing mechanical stimulation per se (Yap et al., 2019). The convergence of these two pathways occurs at the level of the mitochondria that are activated by both mechanotransduction and magnetoreception and synergize at the level of ROS-dependent feedback activation of TRPC1 (Gervasio et al., 2008). Evidence also exists indicating that the micromechanical environment acts synergistically with magnetic fields to better promote cellular development (Celik et al., 2020; Parate et al., 2020). Thus, the fact that 2DG is capable of synergizing with brief PEMF stimulation to accentuate hDPSC-derived neurogenic differentiation is in alignment with the sum of these previous findings.

hDPSCs induced into neurogenesis on 2DG exhibit increased expression of ChAT and VAChT (Fig. 1b,c), required for the synthesis of acetylcholine (Kratsios et al., 2015; Ohno et al., 2001; Shen et al., 2011) and its subsequent transport into secretory vesicles (Erickson and Varoqui, 2000). Accordingly, there was more cholinergic-stimulated calcium influx in hDPSCs differentiated on 2DG (Fig. 1d). Next, the synergy between PEMF exposure and 2DG was demonstrated with more choline release (Fig. 4 b) as well as accentuated PCNA, NF-M, and HB-9 expressions (Fig. $3)$, relative to hDPSCs on glass exposed to PEMFs. PCNA levels reflect progenitor cell proliferation leading to differentiation as well as upregulation of neurofilament proteins (such as NF-M), characterize mature neurons, and are responsible for axonal growth and extension (UniProtConsortium, 2016). Moreover, HB-9 is involved in the consolidation of the post-mitotic neuronal phenotype (Arber et al., 1999). The expression levels of PCNA, NF-M, and $H B-9$ were all elevated by PEMF exposure in hDPSCs differentiated on 2DG, relative to glass. These results confirm that the combination of 2DG and PEMFs enhanced neuronal differentiation.

hDPSCs induced into neurogenesis on 2DG exhibited higher expression levels of TRPC1, TRPC6, TRPV1, and TRPM8 $1 \mathrm{~d}$ after neurogenic induction, relative to glass controls (Fig. 2). The elevated expressions of TRP channels on 2DG at this precise developmental stage indicated that hDPSCs would be best poised for TRP-mediated neurogenic activation on the first day after chemical induction, a strategy that has been borne out in previous studies using identical magnetic paradigms (Celik et al., 2020; Parate et al., 2017; Yap et al., 2019). TRPC1 and TRPC6 support $\mathrm{Ca}^{2+}$ entry, which is crucial for neuronal proliferation (Abramowitz and Birnbaumer, 2009), while TRPV1-mediated $\mathrm{Ca}^{2+}$ entry promotes proliferation as well as neuronal excitability and synaptic plasticity (Amantini et al., 2009). TRPM8 is a calcium-permeable cold-sensor that is also implicated in supporting proliferation and differentiation (Bidaux et al., 2012; 2015). Significantly, TRPC1 was the most highly expressed of all the TRP channels examined on the first day after neurogenic induction and was further augmented by growth on 2DG. Despite TRPC1 and TRPM7 being the most ubiquitously expressed of all TRP channels (Jang et al., 2012), genetic silencing of TRPC1, but not TRPM7, precluded response to PEMF exposure (Yap et al., 2019). Moreover, vesicular delivery of TRPC1 is necessary and sufficient to restore responsiveness to magnetic field exposure in TRPC1 knock-down muscle cells (Kurth et al., 2020). Accordingly, adjusting the timing of PEMF exposure to coincide with the developmental window when TRPC1 was most prominent gave the greatest enhancement in hDPSC-derived neurogenesis and was preferentially conferred by 2DG substrates. 
Brief PEMF exposure activates TRPC1-mediated calcium entry, which stimulates mitochondrial respiration and consequent ROS production (Yap et al., 2019). PEMF exposure has been associated with ROS and superoxide production in a variety of cell types including neurons, ultimately enhancing antioxidant defenses as well as stimulating proliferation and differentiation (Ehnert et al., 2017; Falone et al., 2016; Yap et al., 2019). ROS and superoxide, although potentially damaging at high levels, at moderate levels prime mitochondrial adaptations that promote neurogenic differentiation and survival (Valero et al., 2012), through a process known as mitohormesis (Ristow and Schmeisser, 2014). PEMF exposure exerts a novel form of magnetic mitohormesis that obeys an electromagnetic efficacy window consisting of a specified amplitude, duration, and frequency of exposure both in vitro (Celik et al., 2020; Parate et al., 2017; Parate et al., 2020; Yap et al., 2019) and in vivo (Tai et al., 2020), whereby lesser or greater exposures were less effective at promoting tissue differentiation. Paralleling these previous in vitro and in vivo studies, the present study showed that PEMF exposure at $2 \mathrm{mT}$ augmented ROS and superoxide levels over $0 \mathrm{mT}$ (basal) and $3 \mathrm{mT}$ levels (Fig. 4c,d). As stem cell activation is the basis of tissue regeneration in the organism, an equivalency is hence observed between the in vitro (Celik et al., 2020; Parate et al., 2017; Parate et al., 2020; Yap et al., 2019) and in vivo (Tai et al., 2020) scenarios, mutually engaging a TRPC1-mitochondrial axis responsive to magnetic field stimulation.

PEMF stimulation collaterally enhances paracrinemediated development (Parate et al., 2020; Tai et al., 2020), which is subject to modulation by the extracellular mechanical environment (Parate et al., 2020). Although numerous scaffold types and biomaterials have been previously tested in the field of dentistry (Galler et al., 2018; Radal et al., 2019) and analogous PEMFs have been studied in the context of MSC-laden scaffolds (Celik et al., 2020), the current study focused on the comparison between 2DG and glass. 2DG per se stimulates paracrine signaling from DPSCs as well as downstream cellular differentiation (Xie et al., 2017a), aligning with evidence that ROS activates the cell secretome to developmental ends (Scheele et al., 2009). Future studies will examine the secretome ramifications of the graphene-PEMF neurogenic paradigm as well as the utility of 3D graphene scaffolds (Li et al., 2013), in conjunction with specified cell alignment and field directionality (Celik et al., 2020), for the further optimization of PEMF-induced neurogenesis.

The present study laid down the foundations for future tissue engineering strategies combining graphene and non-invasive PEMF exposure for the synergistic enhancement of neurogenic differentiation. Scaffolds made of pristine graphene are cytocompatible and capable of enhancing in vitro and in vivo differentiation of hDPSCs into either neuronal (Li et al., 2013; Tasnim et al., 2018) or osteogenic (Xie et al., 2015; 2019) lineages. Moreover, the neurogenic potential of DPSCs extends beyond the field of dentistry (Ellis et al., 2014; Lou et al., 2018; Sasaki et al., 2011; Yang et al., 2017). Due to its unique electrical properties, graphene has been used in the elaboration of brain sensors (Masvidal-Codina et al., 2019) and scaffolds favoring neuronal regeneration (Akhavan, 2016). Graphene films are also capable of promoting osteogenic differentiation of DPSCs in vitro as well as bone formation on titanium implants (Dubey et al., 2020; Xie et al., 2015; Xie et al., 2017b). Unfortunately, the odontogenic differentiation potential of graphene has yet to be realized (Xie et al., 2017b). Therefore, minimally, graphene films in combination with targeted PEMF treatment can be used to enhance the neurogenic potential of DPSCs in vitro. Potentially, employing an external PEMF source to promote the in vivo implant integration and neurogenic/osteogenic differentiation of autologous hDPSCs within graphene scaffolds appears to be practically exploitable.

Historically, neurogenic induction from progenitor cell classes has proven troublesome and costly. The present study outlined an in vitro regenerative strategy to enhance neurogenic induction by: 1) exploiting the multipotency and ease of isolation of hDPSCs; 2) employing graphene to create a more conducive physical environment with which PEMFs to promote neurogenesis; 3 ) targeting a developmental window wherein TRPC 1 is most highly expressed during early neurogenesis; 4) excluding the use of aminoglycoside antibiotics during hDPSC culture to allow magnetic field treatment to promote neurogenesis; 5) assuring that hDPSCs were not too confluent at the time of magnetic exposure to guarantee the best response to magnetic field exposure.

\section{Conclusions}

Differentiating neurons from stem cells is a challenging, costly, and time-consuming process. The development of platforms that are capable of more rapidly and effectively deriving neurons from hDPSCs will spur advances in tissue engineering strategies aimed at regenerating an innervated dental pulp. Both graphene and PEMFs have been independently shown to promote DPSC-derived neurogenesis. Nonetheless, their combination exceeded the sum of their individual benefits. Graphene also accentuated the expression of TRPC1 during the early stages of neuronal differentiation, establishing a window of opportunity to selectively target TRPC1 with unprecedentedly brief PEMF exposure. Timing PEMF exposure to coincide with the highest 2DG-induced expression of TRPC1 accentuated the expression of neurogenic genes and proteins as well as allowed for a robust PEMFinduced response in cellular respiration, which is a requisite for magnetic mitohormetic developmental induction (Tai et al., 2020; Yap et al., 2019). Thus, such a combinational strategy holds promise for the 
regeneration of an innervated dental pulp for future clinical application.

\section{Acknowledgments}

The authors acknowledge Zac Goh (iHealthtech, National University of Singapore) for the design of the graphical abstract.

VR is supported by grants from the Singapore ministry of Education, Singapore (Academic Research Fund Tier 1, R-221-000-104-114, R-221-000132-114). A.F.-O. would like to acknowledge financial support from the Lee Foundation, Singapore (R-176000-243-731), and the Institute for Health Innovation \& Technology, iHealthtech, at the National University of Singapore.

A.F.-O. is an inventor on patent WO 2019/17863 A1, System, and Method for Applying Pulsed Electromagnetic Fields as well as is a contributor to QuantumTx Pte. Ltd., which elaborates electromagnetic field devices for human use. All other authors declare no conflicts of interest.

\section{References}

Abramowitz J, Birnbaumer L (2009) Physiology and pathophysiology of canonical transient receptor potential channels. FASEB J 23: 297-328.

Akhavan O (2016) Graphene scaffolds in progressive nanotechnology/stem cell-based tissue engineering of the nervous system. J Mater Chem B 4: 3169-3190.

Amantini C, Ballarini P, Caprodossi S, Nabissi M, Morelli MB, Lucciarini R, Cardarelli MA, Mammana G, Santoni G (2009) Triggering of transient receptor potential vanilloid type 1 (TRPV1) by capsaicin induces Fas/CD95-mediated apoptosis of urothelial cancer cells in an ATM-dependent manner. Carcinogenesis 30: 1320-1329.

Arber S, Han B, Mendelsohn M, Smith M, Jessell TM, Sockanathan S (1999) Requirement for the homeobox gene $\mathrm{Hb} 9$ in the consolidation of motor neuron identity. Neuron 23: 659-674.

Asrican B, Paez-Gonzalez P, Erb J, Kuo CT (2016) Cholinergic circuit control of postnatal neurogenesis. Neurogenesis (Austin) 3: e1127310. DOI: $10.1080 / 23262133.2015 .1127310$.

Bahrami N, Bayat M, Mohamadnia A, Khakbiz M, Yazdankhah M, Ai J, Ebrahimi-Barough S (2017) Purmorphamine as a Shh signaling activator small molecule promotes motor neuron differentiation of mesenchymal stem cells cultured on nanofibrous PCL scaffold. Mol Neurobiol 54: 5668-5675.

Bidaux G, Beck B, Zholos A, Gordienko D, Lemonnier L, Flourakis M, Roudbaraki M, Borowiec A-S, Fernández J, Delcourt P (2012) Regulation of activity of transient receptor potential melastatin 8 (TRPM8) channel by its short isoforms. J Biol Chem 287: 2948-2962.
Bidaux G, Borowiec A-s, Gordienko D, Beck B, Shapovalov GG, Lemonnier L, Flourakis M, Vandenberghe M, Slomianny C, Dewailly E (2015) Epidermal TRPM8 channel isoform controls the balance between keratinocyte proliferation and differentiation in a cold-dependent manner. Proc Natl Acad Sci U S A 112: E3345-E3354.

Byers MR, Suzuki H, Maeda T (2003) Dental neuroplasticity, neuro-pulpal interactions, and nerve regeneration. Microsc Res Tech 60: 503-515.

Celik C, Franco-Obregón A, Lee EH, Hui JHP, Yang Z (2020) Directionalities of magnetic fields and topographic scaffolds synergise to enhance MSC chondrogenesis. Acta Biomaterialia 119: 169-183.

Chang C-C, Chang K-C, Tsai S-J, Chang H-H, Lin C-P (2014) Neurogenic differentiation of dental pulp stem cells to neuron-like cells in dopaminergic and motor neuronal inductive media. J Formos Med Assoc 113: 956-965.

Clapham DE (2003) TRP channels as cellular sensors. Nature 426: 517-524.

Crocetti S, Beyer C, Schade G, Egli M, Fröhlich J, Franco-Obregón A (2013) Low intensity and frequency pulsed electromagnetic fields selectively impair breast cancer cell viability. PLoS One 8: e72944. DOI: 10.1371/journal.pone.0072944.

Crocetti S, Beyer C, Unternährer S, Benavides Damm T, Schade-Kampmann G, Hebeisen M, Di Berardino M, Fröhlich J, Franco-Obregón A (2014) Impedance flow cytometry gauges proliferative capacity by detecting TRPC1 expression. Cytometry Part A 85: 525-536.

Dubey N, Morin JLP, Luong-Van EK, Agarwalla SV, Silikas N, Castro Neto AH, Rosa V (2020) Osteogenic potential of graphene coated titanium is independent of transfer technique. Materialia 9. DOI: 10.1016/j.mtla.2020.100604.

Ebrahimi-Barough S, Norouzi Javidan A, Saberi H, Joghataei MT, Rahbarghazi R, Mirzaei E, Faghihi F, Shirian S, Ai A, Ai J (2015) Evaluation of motor neuron-like cell differentiation of hEnSCs on biodegradable PLGA nanofiber scaffolds. Mol Neurobiol 52: 1704-1713.

Ehnert S, Fentz A-K, Schreiner A, Birk J, Wilbrand B, Ziegler P, Reumann MK, Wang H, Falldorf K, Nussler AK (2017) Extremely low frequency pulsed electromagnetic fields cause antioxidative defense mechanisms in human osteoblasts via induction of - $\mathrm{O}_{2}$ - and $\mathrm{H}_{2} \mathrm{O}_{2}$. Scientific Reports 7: 14544. DOI: 10.1038/s41598-017-14983-9.

Ellis KM, O'Carroll DC, Lewis MD, Rychkov GY, Koblar SA (2014) Neurogenic potential of dental pulp stem cells isolated from murine incisors. Stem Cell Res Ther 5: 30. DOI: 10.1186/scrt419.

Engler AJ, Sen S, Sweeney HL, Discher DE (2006) Matrix elasticity directs stem cell lineage specification. Cell 126: 677-689.

Erickson JD, Varoqui H (2000) Molecular analysis of vesicular amine transporter function and targeting to secretory organelles. FASEB J 14: 2450-2458. 
Falone S, Marchesi N, Osera C, Fassina L, Comincini S, Amadio M, Pascale A (2016) Pulsed electromagnetic field (PEMF) prevents pro-oxidant effects of $\mathrm{H}_{2} \mathrm{O}_{2}$ in SK-N-BE(2) human neuroblastoma cells. Int J Radiat Biol 92: 281-286.

Fričová D, Korchak JA, Zubair AC (2020) Challenges and translational considerations of mesenchymal stem/stromal cell therapy for Parkinson's disease. NPJ Regen Med 5: 20. DOI: 10.1038/s41536-020-00106-y.

Galler KM, Brandl FP, Kirchhof S, Widbiller M, Eidt A, Buchalla W, Göpferich A, Schmalz G (2018) Suitability of different natural and synthetic biomaterials for dental pulp tissue engineering. Tissue Eng Part A 24: 234-244.

Gervasio OL, Whitehead NP, Yeung EW, Phillips WD, Allen DG (2008) TRPC1 binds to caveolin-3 and is regulated by Src kinase - role in Duchenne muscular dystrophy. J Cell Sci 121: 2246-2255.

Golovina VA, Platoshyn O, Bailey CL, Wang J, Limsuwan A, Sweeney M, Rubin LJ, Yuan JX-J (2001) Upregulated TRP and enhanced capacitative Ca2+ entry in human pulmonary artery myocytes during proliferation. Am J Physiol Heart Circ Physiol 280: H746-H755.

Gronthos S, Mankani M, Brahim J, Robey PG, Shi S (2000) Postnatal human dental pulp stem cells (DPSCs) in vitro and in vivo. Proc Natl Acad Sci U S A 97: 13625-13630.

Guo R, Zhang S, Xiao M, Qian F, He Z, Li D, Zhang X, Li H, Yang X, Wang M (2016) Accelerating bioelectric functional development of neural stem cells by graphene coupling: implications for neural interfacing with conductive materials. Biomaterials 106: 193-204.

Jang Y, Lee Y, Kim SM, Yang YD, Jung J, Oh U (2012) Quantitative analysis of TRP channel genes in mouse organs. Arch Pharm Res 35: 1823-1830.

Kalbacova M, Broz A, Kong J, Kalbac M (2010) Graphene substrates promote adherence of human osteoblasts and mesenchymal stromal cells. Carbon 48: 4323-4329.

Kim J, Park S, Kim YJ, Jeon CS, Lim KT, Seonwoo $\mathrm{H}$, Cho S-P, Chung TD, Choung P-H, Choung Y-H (2015) Monolayer graphene-directed growth and neuronal differentiation of mesenchymal stem cells. J Biomed Nanotechnol 11: 2024-2033.

Kratsios P, Pinan-Lucarré B, Kerk Sze Y, Weinreb A, Bessereau J-L, Hobert O (2015) Transcriptional coordination of synaptogenesis and neurotransmitter signaling. Curr Biol 25: 1282-1295.

Kurth F, Tai YK, Parate D, van Oostrum M, Schmid YRF, Toh SJ, Yap JLY, Wollscheid B, Othman A, Dittrich PS, Franco-Obregon A (2020) Cell-derived vesicles as TRPC1 channel delivery systems for the recovery of cellular respiratory and proliferative capacities. Adv Biosyst 4: e2000146. DOI: 10.1002/ adbi.202000146.

Lee WC, Lim CHY, Shi H, Tang LA, Wang Y, Lim CT, Loh KP (2011) Origin of enhanced stem cell growth and differentiation on graphene and graphene oxide. ACS Nano 5: 7334-7341.

Lee YJ, Seo TH, Lee S, Jang W, Kim MJ, Sung JS (2018) Neuronal differentiation of human mesenchymal stem cells in response to the domain size of graphene substrates. J Biomed Mater Res A 106: 43-51.

Li J, Wang G, Geng H, Zhu H, Zhang M, Di Z, Liu X, Chu PK, Wang X (2015) CVD growth of graphene on NiTi alloy for enhanced biological activity. ACS Appl Mater Interfaces 7: 19876-19881.

Li N, Zhang Q, Gao S, Song Q, Huang R, Wang L, Liu L, Dai J, Tang M, Cheng G (2013) Threedimensional graphene foam as a biocompatible and conductive scaffold for neural stem cells. Sci Rep 2013: 1604. DOI: 10.1038/srep01604.

Li XJ, Hu BY, Jones SA, Zhang YS, LaVaute T, Du ZW, Zhang SC (2008) Directed differentiation of ventral spinal progenitors and motor neurons from human embryonic stem cells by small molecules. Stem Cells 26: 886-893.

Li Y, Liu Y, Fu Y, Wei T, Le Guyader L, Gao G, Liu R-S, Chang Y-Z, Chen C (2012) The triggering of apoptosis in macrophages by pristine graphene through the MAPK and TGF-beta signaling pathways. Biomaterials 33: 402-411.

Louis M, Zanou N, Van Schoor M, Gailly P (2008) TRPC1 regulates skeletal myoblast migration and differentiation. J Cell Sci 121: 3951-3959.

Luo L, He Y, Wang X, Key B, Lee BH, Li H, Ye $Q$ (2018) Potential roles of dental pulp stem cells in neural regeneration and repair. Stem Cells Int 7: 1731289. DOI: 10.1155/2018/1731289.

Luong-Van EK, Madanagopal TT, Rosa V (2020) Mechanisms of graphene influence on cell differentiation. Mater Today Chem 16. DOI: 10.1016/j. mtchem.2020.100250.

Ma Q, Chen C, Deng P, Zhu G, Lin N, Zhang L, Xu S, M H, Lu Y, Duan W, Pi H, Cao Z, Pei L, Li M, Liu C, Zhang Y, Zhong M, Zhou Z, Yu Z (2016) Extremely low-frequency electromagnetic fields promote in vitro neuronal differentiation and neurite outgrowth of embryonic neural stem cells via up-regulating TRPC1. PloS One 11. e0150923. DOI: 10.1371/journal. pone.0150923.

Madanagopal TT, Franco-Obregón A, Rosa V (2020) Comparative study of xeno-free induction protocols for neural differentiation of human dental pulp stem cells in vitro. Arch Oral Biol 109: 104572. DOI: 10.1016/j.archoralbio.2019.104572.

Madhvi A, Hingane S, Srivastav R, Joshi N, Subramani C, Muthumohan R, Khasa R, Varshney S, Kalia M, Vrati S (2017) A screen for novel hepatitis $C$ virus RdRp inhibitor identifies a broad-spectrum antiviral compound. Sci Rep 7: 5816. DOI: 10.1038/ s41598-017-04449-3.

Martens W, Wolfs E, Struys T, Politis C, Bronckaers A, Lambrichts I (2012) Expression pattern of basal markers in human dental pulp stem cells and tissue. Cells Tissues Organs 196: 490-500. 
Masvidal-Codina E, Illa X, Dasilva M, Calia AB, Dragojević T, Vidal-Rosas EE, Prats-Alfonso E, Martínez-Aguilar J, De la Cruz JM, Garcia-Cortadella R, Godignon P, Rius G, Camassa A, Del Corro E, Bousquet J, Hébert C, Durduran T, Villa R, SanchezVives MV, Garrido JA, Guimerà-Brunet A (2019) High-resolution mapping of infraslow cortical brain activity enabled by graphene microtransistors. Nat Mater 18: 280-288.

Matsumura CY, Taniguti AP, Pertille A, Santo Neto H, Marques MJ (2011). Stretch-activated calcium channel protein TRPC1 is correlated with the different degrees of the dystrophic phenotype in mdx mice. Am J Physiol Cell Physiol 301: C1344-C1350.

Morin JLP, Dubey N, Decroix FED, Luong-Van EK, Castro Neto AH, Rosa V (2017) Graphene transfer to 3-dimensional surfaces: a vacuum-assisted dry transfer method. 2D Mater 4: 025060.

Moussa DG, Aparicio C (2018) Present and future of tissue engineering scaffolds for dentin-pulp complex regeneration. J Tissue Eng Regen Med 13: 58-75.

O'Brien FJ (2011) Biomaterials \& scaffolds for tissue engineering. Mater Today 14: 88-95.

Ohno K, Tsujino A, Brengman JM, Harper CM, Bajzer Z, Udd B, Beyring R, Robb S, Kirkham FJ, Engel AG (2001) Choline acetyltransferase mutations cause myasthenic syndrome associated with episodic apnea in humans. Proc Natl Acad Sci U S A 98: 2017-2022.

Papaccio G, Urrutia DN, Caviedes P, Mardones R, Minguell JJ, Vega-Letter AM, et al. (2019) Comparative study of the neural differentiation capacity of mesenchymal stromal cells from different tissue sources: an approach for their use in neural regeneration therapies. PLoS One 14: e0213032. DOI: 10.1371/journal.pone.0213032.

Parate D, Kadir ND, Celik C, Lee EH, Hui JHP, Franco-Obregón A, Yang Z (2020) Pulsed electromagnetic fields potentiate the paracrine function of mesenchymal stem cells for cartilage regeneration. Stem Cell Res Ther 11: 46. DOI: 10.1186/ s13287-020-1566-5.

Parate D, Franco-Obregón A, Fröhlich J, Beyer C, Abbas AA, Kamarul T, Hui JH, Yang Z (2017) Enhancement of mesenchymal stem cell chondrogenesis with short-term low intensity pulsed electromagnetic fields. Sci Rep 7: 9421. DOI: 10.1038/ s41598-017-09892-w.

Park SY, Park J, Sim SH, Sung MG, Kim KS, Hong BH, Hong S (2011) Enhanced differentiation of human neural stem cells into neurons on graphene. Adv Mater 23: H263-H267.

Peccoud J, Peerani R, Onishi K, Mahdavi A, Kumacheva E, Zandstra PW (2009) Manipulation of signaling thresholds in "engineered stem cell niches" identifies design criteria for pluripotent stem cell screens. PLoS One 4: e6438. DOI: 10.1371/journal. pone.0006438.

Raddall G, Mello I, Leung BM (2019) Biomaterials and scaffold design strategies for regenerative endodontic therapy. Front Bioeng Biotechnol 7: 317. DOI: 10.3389/fbioe.2019.00317.

Ristow M, Schmeisser K (2014) Mitohormesis: promoting health and lifespan by increased levels of reactive oxygen species (ROS). DoseResponse 12: 288-341.

Rosa V, Botero TM, Nör JE (2011) Regenerative endodontics in light of the stem cell paradigm. Int Dent J 61: 23-28.

Rosa V, Della Bona A, Cavalcanti BN, Nör JE (2012) Tissue engineering: from research to dental clinics. Dent Mater 28: 341-348.

Rosa V, Zhang Z, Grande RH, Nor JE (2013) Dental pulp tissue engineering in full-length human root canals. J Dent Res 92: 970-975.

Rosa V, Xie H, Dubey N, Madanagopal TT, Rajan SS, Morin JLP, Islam I, Castro Neto AH (2016) Graphene oxide-based substrate: physical and surface characterization, cytocompatibility and differentiation potential of dental pulp stem cells. Dent Mater 32: 1019-1025.

Sakai VT, Zhang Z, Dong Z, Neiva KG, Machado MA, Shi S, Santos CF, Nor JE (2010) SHED differentiate into functional odontoblasts and endothelium. J Dent Res 89: 791-796.

Samiei M, Aghazadeh Z, Abdolahinia ED, Vahdati A, Daneshvar S, Noghani A (2020) The effect of electromagnetic fields on survival and proliferation rate of dental pulp stem cells. Acta Odontol Scand 78: 494-500.

Sasaki R, Aoki S, Yamato M, Uchiyama H, Wada K, Ogiuchi H, Okano T, Ando T. (2011) PLGA artificial nerve conduits with dental pulp cells promote facial nerve regeneration. J Tissue Eng Regen Med 5: 823830.

Scheele C, Nielsen S, Pedersen BK (2009) ROS and myokines promote muscle adaptation to exercise. Trends Endocrinol Metab 20: 95-99.

Shen XM, Crawford TO, Brengman J, Acsadi G, Iannaconne S, Karaca E, Khoury C, Mah JK, Edvardson S, Bajzer Z (2011) Functional consequences and structural interpretation of mutations of human choline acetyltransferase. Hum Mutat 32: 1259-1267.

Shi S, Robey PG, Gronthos S (2001) Comparison of human dental pulp and bone marrow stromal stem cells by cDNA microarray analysis. Bone 29: 532-539.

Tai YK, Ng C, Purnamawati K, Yap JLY, Yin JN, Wong C, Patel BK, Soong PL, Pelczar P, Fröhlich J, Beyer C, Fong CHH, Ramanan S, Casarosa M, Cerrato CP, Foo ZL, Selvan RMP, Grishina E, Degirmenci U, Toh SJ, Richards PJ, Mirsaidi A, Wuertz-Kozak K, Chong SY, Ferguson SJ, Aguzzi A, Monici M, Sun L, Drum CL, Wang JW, Franco-Obregón A (2020) Magnetic fields modulate metabolism and gut microbiome in correlation with PGC- $1 \alpha$ expression: follow-up to an in vitro magnetic mitohormetic study. FASEB J 34: 11143-11167.

Tasnim N, Thakur V, Chattopadhyay M, Joddar B. (2018) The efficacy of graphene foams for culturing mesenchymal stem cells and their differentiation into 
dopaminergic neurons. Stem Cells Int 2018: 3410168. DOI: $10.1155 / 2018 / 3410168$.

Torossian F, Bisson A, Vannier J-P, Boyer O, Lamacz M (2010) TRPC expression in mesenchymal stem cells. Cell Mol Biol Lett 15: 600. DOI: 10.2478/ s11658-010-0031-3.

Toth AB, Shum AK, Prakriya M (2016) Regulation of neurogenesis by calcium signaling. Cell Calcium 59: 124-134.

UniProtConsortium (2016) Neurofilamentmedium, UniProtKB protein knowledgebase.

Valero T, Moschopoulou G, Mayor-Lopez L, Kintzios S (2012) Moderate superoxide production is an early promoter of mitochondrial biogenesis in differentiating N2a neuroblastoma cells. Neurochem Int 61: 1333-1343.

Wada T, Honda M, M inami I, Tooi N, Amagai Y, Nakatsuji N, Aiba K (2009) Highly efficient differentiation and enrichment of spinal motor neurons derived from human and monkey embryonic stem cells. PLoS One 4: e6722. DOI: 10.1371/journal. pone.0006722.

Wang YK, Yu X, Cohen DM, Wozniak MA, Yang MT, Gao L, Eyckmans J, Chen CS (2012) Bone morphogenetic protein-2-induced signaling and osteogenesis is regulated by cell shape, RhoA/ROCK, and cytoskeletal tension. Stem Cells Dev 21: 11761186.

Xie H, Cao T, Franco-Obregón A, Rosa V (2019) Graphene-induced osteogenic differentiation is mediated by the integrin/FAK axis. Int J Mol Sci 20: 574. DOI: 10.3390/ijms20030574.

Xie H, Cao T, Gomes JV, Castro Neto AH, Rosa V (2015) Two and three-dimensional graphene substrates to magnify osteogenic differentiation of periodontal ligament stem cells. Carbon 93: 266-275.

Xie H, Dubey N, Shim W, Ramachandra CJA, Min KS, Cao T, Rosa V (2017) Functional odontoblasticlike cells derived from human iPSCs. J Dent Res 97: 77-83.

Xie H, Chua M, Islam I, Bentini R, Cao T, VianaGomes JC, Castro Neto AH, Rosa V (2017) CVDgrown monolayer graphene induces osteogenic but not odontoblastic differentiation of dental pulp stem cells. Dent Mater 33: e13-e21.

Xuan K, Li B, Guo H, Sun W, Kou X, He X, Zhang Y, Sun J, Liu A, Liao L, Liu S, Liu W, Hu C, Shi S, Jin Y (2018) Deciduous autologous tooth stem cells regenerate dental pulp after implantation into injured teeth. Sci Transl Med 10: eaaf3227. DOI: 10.1126/ scitranslmed.aaf3227.

Yap JLY, Tai YK, Fröhlich J, Fong CHH, Yin JN, Foo ZL, Ramanan S, Beyer C, Toh SJ, Casarosa M, Bharathy N, Kala MP, Egli M, Taneja R, Lee CN, Franco-Obregón A (2019) Ambient and supplemental magnetic fields promote myogenesis via a TRPC1-mitochondrial axis: evidence of a magnetic mitohormetic mechanism. FASEB J 33: 12853-12872.

Yang C, Li X, Sun L, Guo W, Tian W (2017) Potential of human dental stem cells in repairing the complete transection of rat spinal cord. J Neural Eng 14: 026005. DOI: 10.1088/1741-2552/aa596b.

\section{Discussion with Reviewers}

Pierfrancesco Pagella: Can the authors explain in more detail how they preconfigure a therapeutic approach using graphene/PEMFs-prestimulated hDPSCs?

Authors: DPSCs can be obtained from a third molar, under local anesthesia. Hence, their ease of isolation makes hDPSCs a promising cell source for autologous cell therapies (Rosa et al., 2012). This study will lay down the foundations for future tissue engineering strategies whereby graphene and non-invasive PEMF exposure can act synergistically to enhance neurogenic differentiation. Remarkably, scaffolds made of pristine graphene are cytocompatible and enhance in vitro and in vivo cell differentiation of hDPSCs into multiple lineages (Xie et al., 2019; 2015), including neurons (Li et al., 2013; Tasnim et al., 2018). Hence, employing an external PEMF source to promote neurogenic differentiation of autologous hDPSCs within graphene scaffolds implanted in vivo is practically exploitable. For instance, to promote the neurogenic differentiation and innervation of craniofacial and dental tissues. Finally, 3D scaffolds can also be generated from graphene with neurogenic potential (Li et al., 2013) that can be ultimately combined with PEMF exposure for dental applications. The present study focused on 2D for its simplicity and minimization of mechanically arising confounding factors.

Editor's note: The Scientific Editor responsible for this paper was Thimios Mitsiadis. 